\title{
Primitive and Point Configuration Texture Model and Primitive Estimation Using Mathematical Morphology
}

\author{
Akira Asano $^{1}$, Takeshi Ohkubo ${ }^{1}$, Mitsuji Muneyasu $^{2}$, and Takao Hinamoto ${ }^{1}$ \\ 1 Hiroshima University, Higashi-Hiroshima, Hiroshima 739-8521 JAPAN, \\ asano@mis.hiroshima-u.ac.jp, \\ http://kuva.mis.hiroshima-u.ac.jp/ \\ 2 Kansai University, Suita, Osaka 564-8680, JAPAN
}

\begin{abstract}
A model for texture description, called "Primitive and Point Configuration (PPC) texture model," and an estimation method of the primitive, which is an elementary object for configuring a texture, are proposed in this paper. The PPC texture model regards that a texture is composed by arranging grains that are derived from one or a few primitives by some modification. The primitive shape is estimated by the principle that the primitive resembling the grains best should be the optimal estimation. This estimation is achieved by finding the structuring element that minimizes the integral of the size distribution function of a target texture.
\end{abstract}

\section{$1 \quad$ PPC Texture Model}

An image is recognized as a texture in case that a lot of simple objects, relatively smaller than the whole image, are arranged regularly or randomly, and the extent of the arrangement seems to be much larger than the actual image size. Various methods of texture analysis, for example the cooccurence matrix method and the spatial frequency method, have been proposed [1]. These methods measure characteristics of a texture by assuming the structure of the repetitive object arrangement whose extent does not depend on the image size. The measurement of texture characteristics is often employed for texture classification and discrimination, which are main topics of image analysis.

Our aim of texture analysis is description, rather than measurement. The texture description analyzes how a texture is generated under a deterministic or statistical model, and presents a method of reorganizing or modifying the texture from the results of analysis. We propose a model of texture description, called "Primitive and Point Configuration (PPC) texture model," and a method of parameter estimation based on the model in this paper. The PPC texture model is also based on the above observation of the texture. It considers a texture to be composed by locating grains on an image plane. A grain is defined as a locally extended figure which appears in an actual texture. We assume that a grain is derived from one or a few primitives by some modification. 
Consequently, a primitive is a model parameter estimated for a texture, and its shape determines local characteristics of a texture. The PPC model is similar to well-known Boolean model [2]. However, the Boolean model is based on the grain location configuration by Poisson random process, while the grains can be located either regularly or randomly, in other words either deterministically or statistically, in the PPC model. The grain location configuration determines global characteristics of a texture. A regular or deterministic configuration produces a regular texture like woven cloths, and a random configuration produces a random texture like rough surfaces on natural materials.

An advantage of the PPC model is independent characterization of primitive shapes and grain configurations. This enables description of primitives independently on the configuration or density of grains. Since this model handles global and local characteristics of a texture independently, it enables flexible modifications of one characteristic independently on the other. These modifications are, for example, applicable to the conversion of random textures to regular ones with the same periodicity for comparison, and the generation of similar textures by parameterizing the random point process of grain location configuration.

\section{Primitive Estimation}

Application of the PPC model to practical texture analysis requires a method of estimating primitive shape and point configuration simultaneously. Note that there are several possible estimations of the pair of primitive and point configuration for a texture. For example, a binary texture image is constructed by onepixel primitive located at every white pixels in the texture, or by a large primitive identical to the texture itself located at the center of the image. Contrarily to these trivial estimations, practically meaningful estimations of the primitive are required to resemble grains appearing repeatedly in a texture. We assume, in order to achieve this, that grains are derived by homothetic magnification from one primitive of limited size. If it is assumed that the primitive shape has been already estimated under the above assumption, the grain location configuration is derived by morphological skeleton [3][4] using the primitive as the structuring element. The morphological skeleton is explained intuitively as follows: Suppose covering the object with preferably large homothetic magnifications of a structuring element in the following way. At first we locate the largest magnification contained within the object, and cover the object by sweeping the magnification within the object. Then gradually smaller magnifications are employed for covering the residual region until the whole object is covered. The skeleton is defined as the union of the origins of all the employed magnifications of the structuring element. The skeleton of the target texture is equivalent to the grain location configuration, since the original texture is reconstructed by locating a correspondingly magnified structuring element at each point of the skeleton.

Since the grain location configuration is derived as explained above, we concentrate upon the estimation of primitive shape in this paper. Examples of estimated grain location configurations and their modification are explained in the 
other paper of ours in this conference [10]. We have already proposed applicable estimation methods based on an assumption on the size density function [5][6] and based on the skeleton [7] in the context of texture characterization. However, the former ones require an assumption on the size distribution of grains, and the latter one causes a faulty result provided that the size limitation of a primitive is large. We propose a novel method based on the size distribution function [8][9] of a texture in this paper. The value of the size distribution function at size $n$ is defined as the ratio of residual area of an object by the morphological opening by the $n$-times magnification of the structuring element to the whole area of the object. The value of size distribution function at size $n$ indicates the relative area of structures that are too small to contain the $n$-times magnification of the structuring element.

The optimal estimation of the primitive of a texture in our criterion is the structuring element minimizing the integral of the size distribution function of the target texture. The reason of employing this criterion is as follows: If the structuring element is homothetic to an object, the size distribution function contains only one peak at the size of the object relative to the original structuring element, and the values of the function at smaller sizes are zero. If the structuring element is nearly homothetic to the object, the openings by the magnifications of small sizes produce some residual structures, and some nonzero values of the function appear at small sizes. Since the optimal estimation of the primitive should resemble the grains appearing in the texture as explained in the above, these observations indicates that the optimal estimation of the primitive is obtained by finding the structuring element that minimizes residual structures, i. e. minimizes the values of the size distribution function at small sizes. Since the size distribution function is monotonically increasing and bounded to unity at a sufficiently large size, this is achieved by minimizing the integral of the size distribution function. Finding the optimal structuring element from a set of structuring elements with a certain flexibility is achieved by the probabilistic optimization using the simulated annealing method.

\section{Method}

We explain the formal definition of the size distribution function, and the whole optimization procedure using the simulated annealing, in the followings.

\subsection{Size distribution function}

The size distribution function of size $n$ relative to the structuring element $B$ for image $X$, denoted as $F(X, B, n)$, is defined as follows:

$$
F(X, B, n)=A\left\{X-X_{n B}\right\},
$$

where $A$ indicates the area of the operand if the operand is a binary image, and the sum of pixel values if the operand is a gray scale one. $X_{B}$ denotes opening of $X$ by $B$, defined as follows: 


$$
X_{B}=(X \ominus \check{B}) \oplus B
$$

and $n B$ is the $n$-times homothetic magnification of $B$. This is usually defined as follows:

$$
\begin{gathered}
n B=B \oplus B \oplus \ldots \oplus B \quad((n-1)-\text { times of } \oplus), \\
0 B=\{0\} .
\end{gathered}
$$

where $\oplus$ denotes the Minkowski set addition. This definition is, however, inconvenient since the difference between $n B$ and $(n+1) B$ is too large if the original $B$ is large. Thus we employ another definition in this paper, as follows:

$$
n B=B \oplus C \oplus \ldots \oplus C \quad((n-1)-\text { times of } \oplus),
$$

where $C$ is another small structuring element. The operation $X-X_{n B}$ is also known as Top-Hat transformation [3].

The integral of the size distribution function, denoted $\operatorname{IF}(X, B, N)$, is defined as follows:

$$
I F(X, B, N)=\sum_{n=0}^{N} F(X, B, n),
$$

where $N$ is the maximum size for calculation.

\subsection{Optimization by simulated annealing}

Our method minimizes the integral of the size distribution function by iterative modifications of the structuring element. We apply the simulated annealing to the optimization. The optimization procedure is as follows:

1. Assigning an initial structuring element to $B$.

2. Deriving the size distribution function $F(X, B, n)$ and the integral $I F(X, B, N)$ by the above procedure.

3. Assigning a modification of $B$ to $B_{i}^{\prime}$, where $i$ is the iteration index.

4. Deriving the size distribution function $F\left(X, B_{i}^{\prime}, n\right)$ and the integral $I F\left(X, B_{i}^{\prime}, N\right)$.

5. Comparing $\operatorname{IF}(X, B, n)$ and $\operatorname{IF}\left(X, B_{i}^{\prime}, N\right)$.

6. - if $I F(X, B, N)>I F\left(X, B_{i}^{\prime}, N\right)$, replacing $B$ with $B_{i}^{\prime}$ and go back to the step 3 ,

- if $I F(X, B, N) \leq I F\left(X, B_{i}^{\prime}, N\right)$, replacing $B$ with $B_{i}^{\prime}$ by a small probability to avoid reaching a local minimum, and then go back to step 3.

7. Iterating steps $3-6$ until the replacement of $B$ with $B_{i}^{\prime}$ does not occur any more or a sufficient number of iteration has been carried out. 
We perform a two-stage optimization; The first stage assumes a binary structuring element, and derives the optimal shape of the structuring element by the above procedure. The second stage optimizes the gray scale pixel values of the structuring element whose shape is obtained at the first stage. Details of the binary and gray scale structuring element modifications in step 3 and the probability in Step 6 are explained in the followings.

Modification of binary structuring element A structuring element used for the skeletonization is regarded as the definition of unit distance in the sense of the distance transformation. The distance between each pixel within the structuring element and the origin is regarded as unity. Thus we modify the structuring element under the condition that it contains the origin and is convex in the sense of 8-pixel neighborhood.

We assume at first a binary structuring element, and assume that the number of pixel composing the structuring element is fixed. The structuring element is modified at an iteration in the following subprocedures:

1. Choosing randomly one pixel of the structuring element, and altering the chosen pixel to zero.

2. Choosing randomly another pixel surrounding the structuring element, and altering the chosen pixel to unity.

3 . If the altered pixel violates the above condition of convexity, the above two alterations are cancelled and the procedure restarts from the former random choosing. Otherwise this alteration is accepted and the resultant structuring element is used as $B_{i}^{\prime}$ in step 3 of the main optimization algorithm.

Modification of gray scale structuring element The gray scale pixel values of the structuring element are modified at an iteration in the following subprocedures:

1. Choosing randomly one pixel of the structuring element.

2. Choosing randomly an integer value from the range $[-\alpha, \alpha]$, where $\alpha$ is a constant positive number.

3. Adding the chosen integer to the value of the chosen pixel.

Probability of the replacement of $\boldsymbol{B}$ with $\boldsymbol{B}_{\boldsymbol{i}}^{\prime}$ In step 6 of the main optimization algorithm, we compare $I F(X, B, N)$ and $I F\left(X, B_{i}^{\prime}, N\right)$, and determine whether the modification of structuring element is accepted or not. If $I F(X, B, N)>I F\left(X, B_{i}^{\prime}, N\right)$, the modification from $B$ to $B_{i}^{\prime}$ is always accepted and $B$ in the next iteration is replaced with the current $B_{i}^{\prime}$. If $\operatorname{IF}(X, B, N) \leq$ $\operatorname{IF}\left(X, B_{i}^{\prime}, N\right)$, the modification is accepted with the probability $P\left(B, B_{i}^{\prime}\right)$, defined as follows:

$$
P\left(B, B_{i}^{\prime}\right)=\frac{1}{1+\exp \left(\frac{I F\left(X, B_{i}^{\prime}, N\right)-I F(X, B, N)}{T(i)}\right)},
$$


where $T(i)$ is called "temperature" at the $i$ th iteration. $T(i)$ decreases along the progress of iteration. If the modification is not accepted, it is cancelled.

\section{Experiment}

We carried out an experiment of the primitive estimation using example textures. We optimized the gray scale structuring element composed with 9 pixels by the algorithm shown above. We fixed the parameter $\alpha=10$, and defined the structuring element $C$ in Eq. 5 as $2 \times 2$-pixel square. Pixel values of $C$ are all 10 for the gray scale case. We defined the temperature parameter $T(i)$ in this case as follows:

$$
T(i)=100 \times 0.98^{(i-1)} .
$$

We terminated the algorithm at 1000 iterations in this experiment because of the restriction of computational costs. We tried the optimization several times for one experiment and selected the best structuring element. We employed a crosslike initial structuring element as shown in Fig. 1 for each optimization. A square corresponds to a white pixel in the structuring element. The structuring elements during the optimization is restricted to be connected and contain exactly 9 white pixels. Gray scale values of the structuring elements are restricted to the range of $0-100$. The constant $N$ in Eq. 6, the largest size for the integration, is set to 5 .

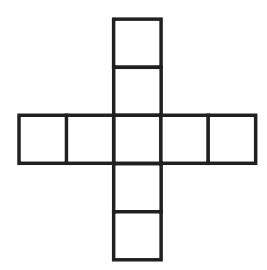

Fig. 1. Initial structuring element.

Figure 2 shows example texture images of $64 \times 64$ pixels[11][12]. Each of texture pairs $\mathrm{A}-\mathrm{A}^{\prime}, \mathrm{B}-\mathrm{B}^{\prime}$, and $\mathrm{C}-\mathrm{C}^{\prime}$ is extracted from different parts of a common texture image. The estimated primitives from these textures are shown in Fig. 3. This result shows that the binary shapes of estimated primitives resemble basic structures of the textures visually well. It also shows that the estimation of the binary shapes is almost stable, i. e. almost the same estimation is obtained from each of the similar texture pairs, and different estimations are obtained from visually different textures. This result shows, however, gray scale values of the estimate primitives are less stable than the binary shapes. Table 1 shows the integrals of the size distribution functions, which are minimized by the optimization algorithm and indicate fitness of the estimation. The fitness is higher for 
the gray scale estimation than for the binary estimation in cases of all textures. These results suggest that the gray scale values obtained by our method are too sensitive to each realization of the randomness of a texture, and further restrictions for the optimization are required to obtain more appropriate modelization of a texture.

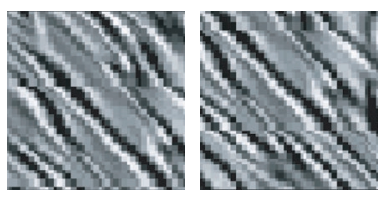

A
$\mathrm{A}^{\prime}$

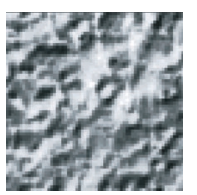

$\mathrm{B}$

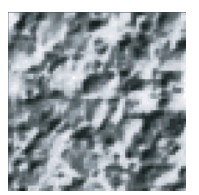

$\mathrm{B}^{\prime}$

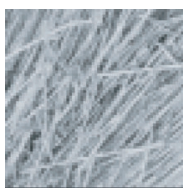

$\mathrm{C}$

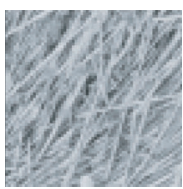

$\mathrm{C}^{\prime}$

Fig. 2. Example textures.

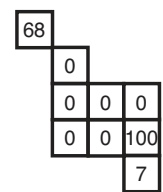

A

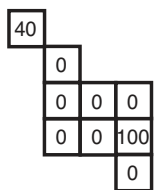

$\mathrm{A}^{\prime}$

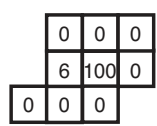

B

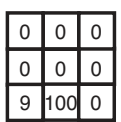

B' $^{\prime}$

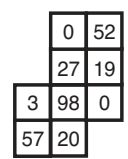

C

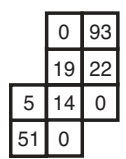

$\mathrm{C}^{\prime}$

Fig. 3. Estimated primitives.

Table 1. Fitness of estimated primitives.

\begin{tabular}{|c|c|c|c|}
\hline Example & initial S.E. & binary estimation & gray scale estimation \\
\hline $\mathrm{A}$ & 1.9611 & 1.5639 & 1.2085 \\
\hline $\mathrm{A}^{\prime}$ & 2.0924 & 1.6452 & 1.2515 \\
\hline $\mathrm{B}$ & 1.9946 & 1.6630 & 1.0975 \\
\hline $\mathrm{B}^{\prime}$ & 2.0774 & 1.7033 & 1.1141 \\
\hline $\mathrm{C}$ & 1.1666 & 1.0129 & 0.7043 \\
\hline $\mathrm{C}^{\prime}$ & 1.1765 & 1.0310 & 0.7178 \\
\hline
\end{tabular}

\section{Conclusions}

We have proposed "Primitive and Point Configuration (PPC)" texture model and a primitive estimation method in this paper. The PPC texture model considers a texture to be composed by locating grains that are derived from one or 
a few primitives. If we assume that the grains are derived from one primitive by a homothetic magnification, the grain location configuration is estimated by the morphological skeleton using the estimated primitive as the structuring element. The primitive is estimated based on the principle that the optimal estimation of the primitive yields the minimum residual structures by openings using homothetic magnifications of the primitive as the structuring elements, and obtained by minimization of the integral of the size distribution function. Experimental results of the estimation of primitives have been shown.

The PPC model characterizes the primitive shape and the grain location configuration separately. This enables characterization of primitives independently on the configuration or density of grains. This also enables modification of the configuration, and assumption of a probabilistic distribution generating the configuration and estimation of the parameters, independently on the primitive shape. For example, a new texture with the same local characteristics as the original one and with different global characteristics is generated by preserving the primitive and replacing the location configuration with a probabilistic process. The deterministic and statistical modifications of the grain location configuration are discussed in [10].

\section{References}

1. T. Ojala and M. Pietikäinen, "Texture classification," in R. B. Fisher, ed., CVonline: The Evolving, Distributed, Non-Proprietary, On-Line Compendium of Computer Vision. (http://www.dai.ed.ac.uk/CVonline/LOCAL_COPIES/ OJALA1/texclas.htm)

2. Serra, J., ed.: Image Analysis and Mathematical Morphology Volume 2: Technical Advances. Academic Press (1988)

3. Serra, J.: Image Analysis and Mathematical Morphology. Academic Press (1982)

4. Maragos, P and R. W. Schafer, R. W.: Morphological skeleton representation and coding of binary image. IEEE Trans. Acoust. Speech Signal Proc. 34 (1986) 12281244

5. Asano, A., Miyagawa, M., and Fujio, M.: Texture Modelling by Optimal Gray Scale Structuring Elements using Morphological Pattern Spectrum. Proc. 15th International Conference on Pattern Recognition 3 (2000) 479-482

6. Asano, A., Miyagawa, M., and Fujio, M.: Morphological texture analysis using optimization of structuring elements. Geometry, Morphology, and Computational Imaging, Springer LNCS 2616 (2003) 141-152

7. Asano, A., Ohkubo, T., Muneyasu, M., and Hinamoto, T.: Texture Primitive Description Using Morphological Skeleton. Proc. International Symposium on Mathematical Morphology VI (2002) 101-108

8. Heijmans, H. J. A. M.: Morphological Image Operators. Academic Press (1994)

9. Maragos, P.: Pattern Spectrum and Multiscale Shape Representation. IEEE Trans. Pattern Anal. Machine Intell. 11 (1989) 701-706

10. Kobayashi, Y. and Asano, A.: Modification of spatial distribution in Primitive and Point Configuration texture model. to be presented in SCIA2003

11. Brodatz, P.: Textures - A Photographic Album for Artists and Designer. Dover, New York (1966)

12. Randen, T.: Brodatz Textures (http://www.ux.his.no/ ${ }^{\sim}$ tranden/brodatz.html) 